\title{
Sleep quality as a mediator of problematic smartphone use and clinical health symptoms
}

\author{
XIAOCHUN XIE ${ }^{1}$, YAN DONG ${ }^{2}$ and JINLIANG WANG ${ }^{3}$ \\ ${ }^{1}$ School of Psychology, Northeast Normal University, Changchun, China \\ ${ }^{2}$ Department of Psychology, The Center of Internet + Social Psychology, Renmin University of China, Beijing, China \\ ${ }^{3}$ Faculty of Psychology, Southwest University, Chongqing, China
}

(Received: December 1, 2017; revised manuscript received: March 30, 2018; second revised manuscript received: April 5, 2018; accepted: April 7, 2018)

\begin{abstract}
Background and aims: Although smartphone use brings many benefits for adolescents, it is also associated with many serious health problems. This study examined the relationship between problematic smartphone use (PSU) and clinical health symptoms (e.g., body dysfunction) and the mediating effects of sleep quality on this relationship in adolescents. Methods: Participants in this cross-sectional survey were 686 middle- and high-school students (girls $=55.7 \%, M_{\text {age }}=12.98 \pm 1.38$ years). Participants completed self-report measures of PSU, sleep quality, and physical symptoms. Correlation analyses and structural equation modeling between adolescents' PSU and the variables of interest were conducted. Results: This study indicated that there was a significant positive correlation between PSU and health symptoms. Furthermore, sleep quality mediated the relationship between PSU and health symptoms. Discussion and conclusions: Findings suggest that to promote health and wellness in adolescents, individuals should be encouraged to place boundaries on smartphone use, especially at bedtime. Reducing adolescents' exposure to smartphone use in this way may hold promise for improving the efficacy of PSU prevention efforts for adolescents.
\end{abstract}

Keywords: problematic smartphone use, sleep quality, clinical health symptoms, adolescents

\section{INTRODUCTION}

A recent survey revealed that an estimated 200 million adolescents in China are smartphone users (China Internet and Information Center, 2015). Smartphones have already become a necessity in the lives of adolescents and create a new and complex virtual context in which youth interact and socialize. Smartphones readily facilitate activities such as online social networking and online gaming. However, problematic smartphone use (PSU) may contribute to the development of health problems, such as depression (Choi, Lee, \& Ha, 2012) and daytime fatigue (Kim, Ahn, Jeon, \& Lee, 2012), and also negatively impact upon academic performance (Liu, Kirschner, \& Karpinski, 2017). As a critical period encompassed by alterations in physical and psychological development (Schulenberg, Maggs, \& Hurrelmann, 1997), adolescence may increase the likelihood of experiencing health hazards. Relatively immature levels of self-regulation make adolescence a time of vulnerability and may lead to a higher likelihood of smartphone addiction and the development of other disorders among adolescents (Bianchi \& Phillips, 2005).

Despite the prevalence of problems associated with smartphone use, relatively few studies have examined the adverse health effects of smartphone use specifically on adolescents (Strasburger, 2009). Moreover, little attention has been given to the potential mechanisms through which smartphone use affects adolescent health, as prior studies have only focused on the direct relationship between smartphone use and adolescent health issues (Demirci, Akgönül, \& Akpinar, 2015). Drawing on recent studies (Li, Lepp, \& Barkley, 2015; Wang, Wang, Gaskin, Wang, 2015) and recurring public concerns, we seek to explore whether PSU may be related to physical and mental health symptoms among Chinese adolescents.

\section{The relationship between PSU and physical health symptoms}

PSU may directly lead to various health symptoms. For example, it has been found that smartphone screens are much more harmful than computer and television screens to eyes and can lead to dry eye syndrome for school children (Moon, Lee, \& Moon, 2014). Ko, Kim, and Woo (2013) found that texting on a smartphone may increase the risk of fatigue in the upper trapezius muscles and cause cumulative

\footnotetext{
* Corresponding author: Yan Dong; Department of Psychology, The Center of Internet + Social Psychology, Renmin University of China, No. 59 Zhongguancun Street, Haidian District, Beijing 100872, China; Phone: +86 1352028 6732; Fax: +86 108250 9716; E-mail: dongpsy@ruc.edu.cn
}

This is an open-access article distributed under the terms of the Creative Commons Attribution-NonCommercial 4.0 International License, which permits unrestricted use, distribution, and reproduction in any medium for non-commercial purposes, provided the original author and source are credited, a link to the CC License is provided, and changes - if any - are indicated. 
trauma disorders. PSU may be characteristic of a sedentary lifestyle, and thus adolescents with PSU may be more likely to suffer from physical symptoms, such as lack of physical energy, physiological dysfunction, and weakened immunity (Cao, Sun, Wan, Hao, \& Tao, 2011). Based on previous studies, we hypothesized that PSU would positively correlate with physical health symptoms (H1).

\section{The relationship between poor sleep quality and physical health symptoms}

Sleep is essential not only for work but also for living (Duran, Matter, Bravo, Moreno, \& Reyes, 2014; Galambos, Vargas Lascano, Howard, \& Maggs, 2013; Magnavita \& Garbarino, 2017). Studies have revealed that poor sleep quality is associated with both poor mental and physical health. In terms of mental health, previous studies provide evidence that poor sleep quality is correlated with depressive symptoms (Park et al., 2010) and poor connection with school (Bao et al., 2018). Park et al. (2010) found that adolescents with poor sleep quality reported high levels of alcohol-use disorder and obsessive-compulsive disorder. Besides mental health aspects, individuals lacking good or sufficient sleep often experience negative feelings about physical fitness. Increasingly, studies are finding that poor quality of sleep is associated with impaired daytime functioning (e.g., Cain \& Gradisar, 2010; Léger et al., 2010), weaker immune system (increased susceptibility to colds, fever, and other illnesses, e.g., Graham \& Streitel, 2010; Lashley, 2003; Prather, Puterman, Eple, \& Dhabhar, 2014), body fatigue (e.g., Dinges et al., 1997; Nagane, Suge, \& Watanabe, 2016), and eye syndromes (e.g., Waller, Bendel, \& Kaplan, 2008). For example, students with poor sleep often feel whole-body fatigue, lack motivation, and desire to rest (Nagane et al., 2016). Furthermore, sleep habits in adolescents are highly likely to be erratic, with a pronounced shift of bedtimes to later in the evening, resulting in sleep deprivation during the school week (Crowley, Acebo, \& Carskadon, 2007; Perkinson-Gloor, Lemola, \& Grob, 2013). Therefore, we hypothesized that reduced sleep quality would positively correlate with physical health symptoms (H2).

\section{The relationship between PSU, poor sleep quality, and physical health symptoms}

There has been a particularly pronounced increase in the use of smartphones in adolescents. The availability of smartphones and the potential to use these in late evening and at bedtime may profoundly change adolescents' sleep patterns. All communication channels are available through a single smartphone now. Calling and texting are available through smarthphones, instant messaging, and social network site. With access to Wi-Fi, smartphones allow peers to communicate through multiple channels after school, regardless of location in the home and including the bedroom. Furthermore, smartphones are comfortable to use while lying in bed to surf the Internet, to play games, or to watch online videos as they are lighter than iPads or other tablets. Thus, smartphones are particularly practical to use when lying in bed or at rest. However, prolonged use of a smartphone can compete with and disturb sleep, resulting in a trend toward insufficient sleep and a downgrade in sleep quality
(Lanaj, Johnson, \& Barnes, 2014). Recent evidence suggests that smartphone use during adolescence is related to later bedtimes, shorter sleep duration, and increased sleep disturbance (Lemola, Perkinson-Gloor, Brand, Dewald-Kaufmann, \& Grob, 2015). In addition to the direct displacement of sleep, presleep smartphone use may also increase cognitive, emotional, and physiological arousal, thereby increasing sleep onset latency and reducing total sleep time (Ivarsson, Anderson, Åkerstedt, \& Lindblad, 2009). Finally, smartphone backlights may have a disruptive effect on circadian rhythms with associated sleep consequences, such as sleeping later than intended and thus reducing overall sleep time (Li et al., 2007). Thus, we predicted that poor sleep quality would mediate the relationship between adolescents' addictive smartphone use and health outcomes. In other words, we hypothesized that the more the adolescents were addicted to a smartphone, the weaker their sleep quality, which in turn, would further increase their daytime fatigue, body dysfunction, and eye problems, and simultaneously lower their immunity to fight diseases. Hence, we predicted that poor sleep quality would mediate the relation between PSU and physical health symptoms (H3).

\section{The present study}

Despite the prevalence of smartphone use and the negative impact on well-being associated with smartphone addiction, few studies to date have investigated the link between PSU and physical health problems (Berolo, Wells, \& Amick, 2011). This study aimed to examine PSU and its associations with sleep quality, body dysfunction, eye symptoms, and fatigue. We used structural equation modeling to determine whether sleep quality mediated the relationship between PSU and health symptoms in a Chinese adolescent population.

\section{METHODS}

\section{Participants and procedure}

This cross-sectional survey was conducted in Shanghai in December 2015 among 11- to 17-year-old middle- and high-school adolescents. Students from one middle school $\left(N=326, M_{\text {age }}=12.98\right.$ years, $\left.S D=0.89\right)$ and one high school $\left(N=562, M_{\text {age }}=15.67\right.$ years, $\left.S D=0.64\right)$ were recruited. A total of 686 participants (77\% smartphone users, $M_{\text {age }}=14.76$ years, $S D=1.38$, females $=55.7 \%$ ) reported carrying and regularly using a smartphone and participated into this study.

Data collection took place in classrooms. Trained researchers supported the participants in taking part in the survey. After signing the consent form, adolescents were invited to fill in the pencil-and-paper questionnaires. All the participants were thanked and debriefed at the end.

\section{Measures}

Problematic smartphone use. Problematic use of smartphone was assessed by a 33-item Chinese inventory, which was adapted from the Smartphone Addiction Inventory (Lin et al., 2014) and the Mobile Phone Addiction Inventory 
(Huang, Wang, Qian, Zhong, \& Tao, 2007). Four subdimensions of PSU were identified by confirmatory factor analysis: overuse (14 items), withdrawal (9 items), compulsive behavior ( 7 items), and disturbances ( 3 items). An example question is "I feel distressed or down once I cease using a smartphone for a certain period of time." The respondents were asked to report how often they experienced obsession, dysfunction, or problems such as phantom vibrations and ringing related to the use of smartphone, on a 4-point Likert scale $(1=$ never to $4=$ always $)$. This inventory had good structural validity, $\chi^{2}(489)=1403.47, p<.001$, comparative fit index $(\mathrm{CFI})=0.97$, Tucker-Lewis index $(\mathrm{TLI})=$ 0.97 , root mean square error of approximation (RMSEA) $[90 \% \mathrm{CI}]=0.05[0.05,0.06]$. Nearly all item loadings were above 0.60 . All four dimensions had satisfactory reliability, $\alpha=.86, .88, .81$, and .77 .

Sleep quality. Sleep quality was measured by eight items adapted from the Pittsburgh Sleep Quality Index (Buysse, Reynolds, Monk, Berman, \& Kupfer, 1989). It consists of six items relating to several domains of sleep patterns in the preceding 1-month period. The components refer to domains, such as subjective sleep quality, sleep duration, sleep latency, and sleep disturbances. Respondents were asked to report how often they experienced sleep problems, such as "difficult to sleep within 30 minutes" on a 4-point Likert scale $(1=$ not during the past month to $4=$ frequently $)$. The scale has high reliability, $\alpha=.80$.

Physical Symptom Inventory. Physical symptoms items were selected from Cornell Medical Index (Brodman, Erdmann, \& Wolff, 1949) and the Multidimensional SubHealth Questionnaire of Adolescents (Tao, Hu, Sun, \& Hao, 2008). The measure is a multidimensional inventory that consists of 26 items in total. Four symptoms were included: eye syndromes (three items, e.g., "dry eyes," $\alpha=.76$ ), body fatigue (six items, e.g., "sleepy in class," $\alpha=.82$ ), physiological dysfunction (eight items, e.g., "waist, shoulders, and neck pain," $\alpha=.87$ ), and weakened immunity (four items, e.g., "sore throat and colds," $\alpha=.81)$. Participants rated all items on a 4-point Likert scale $(1=$ not during the past month to $4=$ frequently).

\section{Data analysis}

Descriptive analyses were conducted using means and standard deviations. Correlation analyses and structural equation modeling were conducted to examine unadjusted relationships between the outcome variable (i.e., adolescent PSU) and variables of interest, such as demographics and smartphone usage of adolescents. Data were analyzed using IBM SPSS Statistics (version 23, Chicago, IL, USA) software. To test the mediation effect of sleep quality, we used Mplus 7.0 (Muthén \& Muthén, 1998-2012).

\section{Ethics}

Institute ethics approval was granted by the university's institutional review board. After the permissions were obtained from schools, students who were eligible to participate were invited to complete the questionnaire. This questionnaire was accompanied by a letter explaining the objectives of the project and instructions for completing the questionnaire.

\section{RESULTS}

\section{Descriptive statistics}

The results showed that adolescents spent $1.24 \pm 1.68 \mathrm{hr}$ per day using smartphones. In the analyses examining associations between PSU and physical symptoms, the four dimensions of PSU predicted higher levels of daytime fatigue, eye syndromes, body dysfunctions, and lower immunity and sleep quality. Here, the average score of the four dimensions was used. The results also showed significant associations between smartphone use (time) and health symptoms, but the strength of the links was notably lower (Table 1).

\section{Mediation analyses}

A focal question in this study was whether PSU influenced students' health by adversely affecting their sleep. This hypothesis was modeled and tested by setting direct paths from PSU to sleep quality, and health symptoms, as well as a mediation pathway through sleep quality to the health symptoms (Figure 1). It should be noted that latent variables were created for the four dimensions of PSU (withdrawal, overuse, compulsive behavior, and disturbances) to filter out measurement errors, while physical health symptoms and sleep quality were considered as manifest variables and the mean scores were used. To better account for the categorical, ordinal nature of the PSU indicators, we used weighted least squares with adjusted mean and variance estimation within Mplus 7.0 (Li, 2015). Standardized estimates of the model path were reported in Figure 1. The data fitted the final model very well, $\chi^{2}(652)=1,604.68, p<.05$, CFI $=$ $0.97, \mathrm{TLI}=0.96$, RMSEA $[90 \% \mathrm{CI}]=0.05[0.04-0.05]$.

A bias-corrected bootstrap confidence interval for the indirect effect is presented in Table 2. The results indicated significant indirect paths, such that participants with higher PSU scores experienced poorer sleep, which in turn predicted stronger symptoms of weakened immune systems, daytime fatigue, body dysfunction, and eye discomfort. It is important to note that sleep quality fully mediated the association between PSU and immune symptoms. The indirect model explained $43.90 \%, 34.62 \%, 33.96 \%$, and all variance of the total effect of PSU on eye symptoms, body dysfunction, daytime fatigue, and immunity symptoms, respectively.

\section{DISCUSSION}

\section{Practical and theoretical implications}

The results demonstrated that there were strong relations between smartphone overuse and physical health problems. Physical symptoms in adolescence might be important signs of physical illnesses, and these should be taken seriously by parents and school health staff. Teenagers tend to use smartphones as a medium for socializing, but overuse can result in spending increasing amounts of time on online activities, leading to eye difficulties, spinal disorders, wrist pain, poor sleep quality, lack of physical energy, attentional 


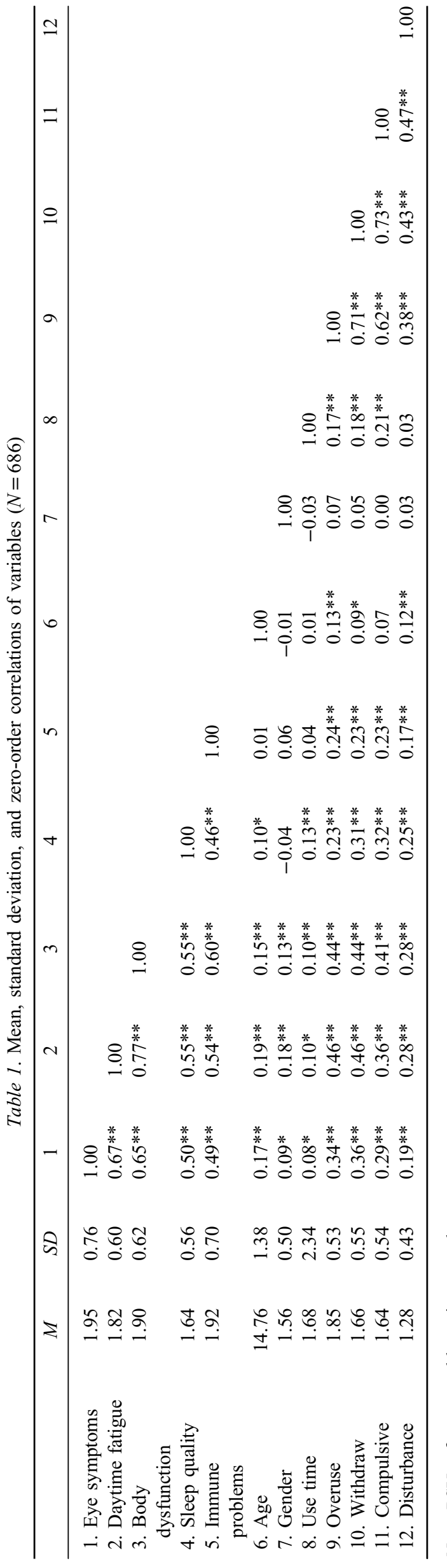

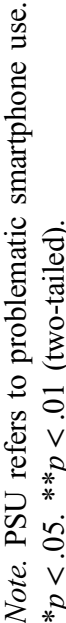

distraction, and immune disorders. The adverse effects of PSU are chronic and progressive, becoming apparent only after months or years, but eventually causing severe health problems. For example, looking down at a smartphone screen to play games, or update Facebook, leads to incrementally increased stresses on the cervical spine (Hansraj, 2014). Moreover, continually stressing the spine may lead to neck pain associated with spine difficulties. Another example is that late night use of a smartphone can cause sleep deprivation and daytime fatigue, which can adversely affect the immune system. Furthermore, smartphone use in darkness is particularly harmful to the eyes. The blue-violet light emitted by smartphones is extremely bright when other lights are off and could have a toxic effect behind the eyes, leading to macular degeneration and eye symptoms (Smick, 2014). As such, PSU may cause chronic problems for adolescents.

The SEM analysis substantiated the hypothesized mediating role of sleep. For adolescents, PSU negatively correlated with physical health, and sleep quality mediated this relation. Establishing healthy sleep routines and controlling smartphone use before and at bedtime are important to facilitate adequate quality sleep and a healthy life.

The present findings may have practical implications for professionals working with clients with PSU. Specifically, these new findings demonstrate that strengthening sleeping quality through reducing PSU before bed may foster physical health and increase immune power among this group of clients. Later bedtimes, shorter sleep duration, and more prolonged sleep latency caused by PSU may lead to chronic health problems in adolescents. Clinicians should be aware of PSU as one potential cause of poor sleep quality when they are designing interventions and care plans for this group. Promoting better sleep routines by minimizing sleep disturbances from PSU could improve body function and school vitality for adolescents.

\section{Limitations and directions for future research}

First, all data collected were self-report, increasing the likelihood of self-report bias. Future studies may use clinical records and sleep diaries to enhance the study validity. Second, the study sample was relatively homogenous (mainly from Shanghai), which limits the generalizability of our findings. Third, most of the variables examined in this study (eye symptoms, body dysfunction, immunity, and fatigue) are physical outcomes and future research may explore the relationship between PSU and mental health symptoms in more depth. Finally, factors such as parental control may moderate the relationship between PSU, sleep quality, and health symptoms, and future research could examine this.

\section{CONCLUSIONS}

In conclusion, although preliminary, our research suggests that PSU is harmful to both mental and physical health. It also emphasizes the importance of sleep quality in 


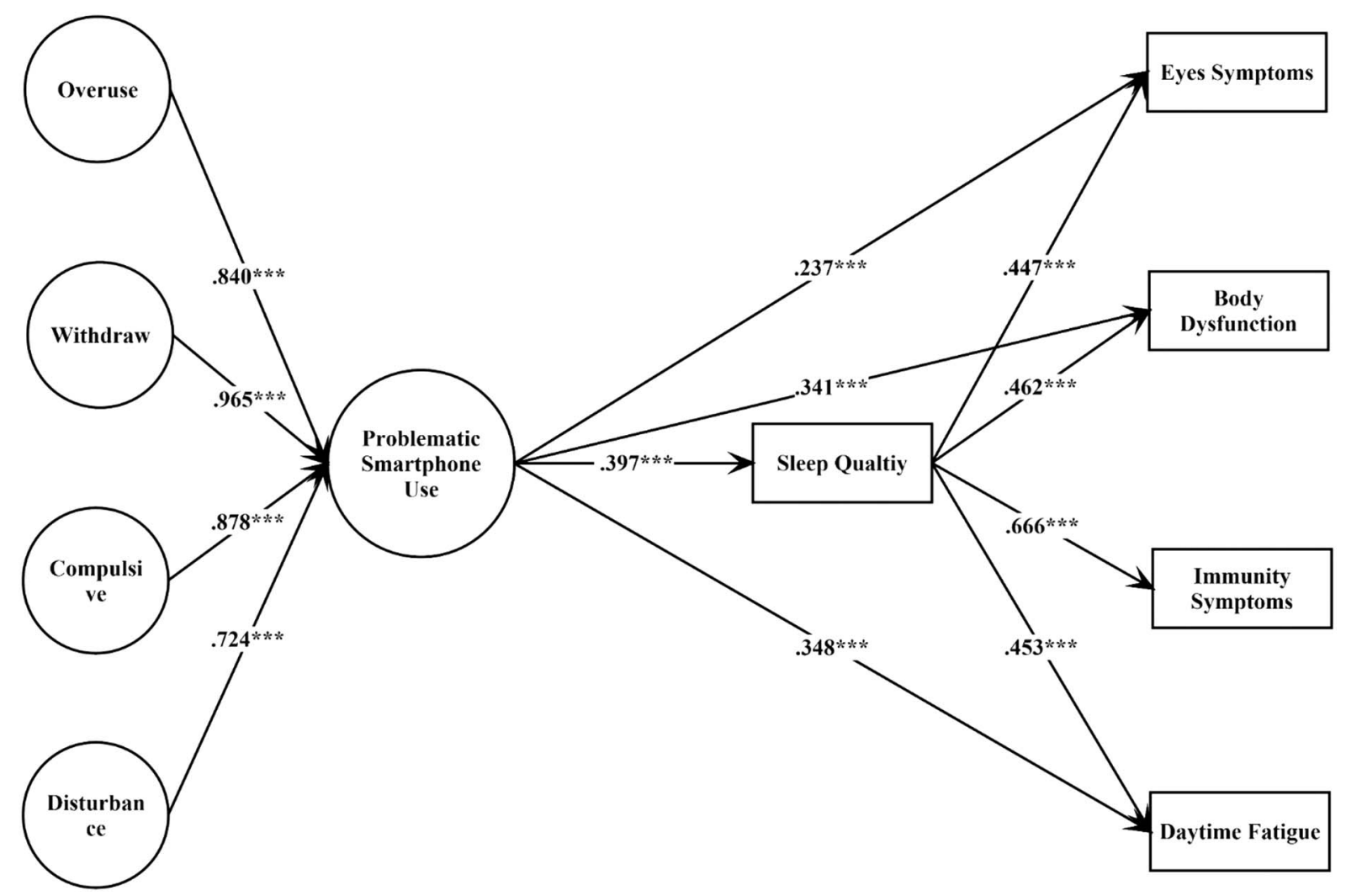

Figure 1. Sleep quality as a mediator between problematic smartphone use and health symptoms

Table 2. Indirect, direct, and total effects of PSU on clinical health symptoms

\begin{tabular}{lcccccc}
\hline Dependent variable & Indirect effect & Boot $S E$ & Boot CI & Direct effect & Total effect & Total effect by indirect (\%) \\
\hline Eye symptoms & $0.18^{* * *}$ & 0.02 & $0.14-0.22$ & $0.24^{* * *}$ & $0.41^{* * *}$ & 43.90 \\
Body dysfunction & $0.18^{* * *}$ & 0.03 & $0.12-0.24$ & $0.34^{* * *}$ & $0.52^{* * *}$ & 34.62 \\
Immunity symptoms & $0.26^{* * *}$ & 0.04 & $0.18-0.34$ & - & $0.26^{* * *}$ & 100 \\
Daytime fatigue & $0.18^{* * *}$ & 0.03 & $0.12-0.24$ & $0.35^{* * *}$ & $0.53^{* * *}$ & 33.96 \\
\hline
\end{tabular}

Note. Total effect by indirect refers to the proportion of indirect effect in total effect. SE: standardized error; CI: confidence interval. $* * * p<.001$ (two-tailed).

mediating the effect of PSU on health symptoms in adolescents. This knowledge is vital for primary school healthcare staff, ophthalmologists, and psychiatrists, so that underlying physical and/or mental problems can be detected and prevented early.

Funding sources: This study was supported by National Natural Science Foundation of China (31500905) and the Fundamental Research Funds for the Central Universities (2412018QD032).

Authors' contribution: XX and YD designed the study, collected and analyzed the data, and wrote the draft. YD also provided the funding sources. YD and JW supervised the study and revised the manuscript. All authors had full access to all data and have taken responsibility for the integrity of the data and the accuracy of the data analysis.
Conflict of interest: There is no conflict of interest and financial conflictions in this study.

Acknowledgements: The authors would like to thank the anonymous the editor and reviewers for their insightful suggestions for the revision of our manuscript.

\section{REFERENCES}

Bao, Z., Chen, C., Zhang, W., Jiang, Y., Zhu, J., \& Lai, X. (2018). School connectedness and Chinese adolescents' sleep problems: A cross-lagged panel analysis. Journal of School Health, 88(4), 315-321. doi:10.1111/josh.12608

Berolo, S., Wells, R. P., \& Amick, B. C. (2011). Musculoskeletal symptoms among mobile hand-held device users and their relationship to device use: A preliminary study in a Canadian 
university population. Applied Ergonomics, 42(2), 371-378. doi:10.1016/j.apergo.2010.08.010

Bianchi, A., \& Phillips, J. G. (2005). Psychological predictors of problem mobile phone use. CyberPsychology \& Behavior, 8(1), 39-51. doi:10.1089/cpb.2005.8.39

Brodman, K., Erdmann, A. J., \& Wolff, H. G. (1949). Cornell Medical Index - Health Questionnaire. Ithaca, NY: Cornell University Medical College.

Buysse, D. J., Reynolds, C. F., Monk, T. H., Berman, S. R., \& Kupfer, D. J. (1989). The Pittsburgh Sleep Quality Index: A new instrument for psychiatric practice and research. Psychiatry Research, 28(2), 193-213. doi:10.1016/0165-1781(89) 90047-4

Cain, N., \& Gradisar, M. (2010). Electronic media use and sleep in school-aged children and adolescents: A review. Sleep Medicine, 11(8), 735-742. doi:10.1016/j.sleep.2010.02.006

Cao, H., Sun, Y., Wan, Y., Hao, J., \& Tao, F. (2011). Problematic Internet use in Chinese adolescents and its relation to psychosomatic symptoms and life satisfaction. BMC Public Health, 11(1), 802. doi:10.1186/1471-2458-11-802

China Internet and Information Center. (2015). Chinese Youth Internet Use in 2014. Retrieved from http://www.cnnic.net.cn/ hlwfzyj/hlwxzbg/qsnbg/201406/P020140611557842544454.pdf

Choi, H. S., Lee, H. K., \& Ha, J. C. (2012). The influence of smartphone addiction on mental health, campus life and personal relations - Focusing on K university students. Journal of the Korean Data and Information Science Society, 23(5), 1005-1015. doi:10.7465/jkdi.2012.23.5.1005

Crowley, S. J., Acebo, C., \& Carskadon, M. A. (2007). Sleep, circadian rhythms, and delayed phase in adolescence. Sleep Medicine, 8(6), 602-612. doi:10.1016/j.sleep.2006.12.002

Demirci, K., Akgönül, M., \& Akpinar, A. (2015). Relationship of smartphone use severity with sleep quality, depression, and anxiety in university students. Journal of Behavioral Addictions, 4(2), 85-92. doi:10.1556/2006.4.2015.010

Dinges, D. F., Pack, F., Williams, K., Gillen, K. A., Powell, J. W., Ott, G. E., Aptowicz, C., \& Pack, A. I. (1997). Cumulative sleepiness, mood disturbance and psychomotor vigilance performance decrements during a week of sleep restricted to 4-5 hours per night. Sleep: Journal of Sleep Research \& Sleep Medicine, 20, 267-277. doi:10.1093/sleep/20.4.267

Duran, A. S., Matter, A. P., Bravo, B. N., Morerno, B. C., \& Reyes, G. S. (2014). Association of quality of life perception with sleeping patterns in Chilean older people. Revista Medica De Chile, 142, 1371-1376. doi:10.4067/S0034-98872014001100002

Galambos, N. L., Vargas Lascano, D. I., Howard, A. L., \& Maggs, J. L. (2013). Who sleeps best? Longitudinal patterns and covariates of change in sleep quantity, quality, and timing across four university years. Behavioral Sleep Medicine, 11(1), 8-22. doi:10.1080/15402002.2011.596234

Graham, J. E., \& Streitel, K. L. (2010). Sleep quality and acute pain severity among young adults with and without chronic pain: The role of biobehavioral factors. Journal of Behavioral Medicine, 33(5), 335-345. doi:10.1007/s10865010-9263-y

Hansraj, K. K. (2014). Assessment of stresses in the cervical spine caused by posture and position of the head. Surgical Technology International, 25, 277-279.

Huang, Z., Wang, M., Qian, M., Zhong, J., \& Tao, R. (2007). Chinese Internet Addiction Inventory: Developing a measure of problematic Internet use for Chinese college students.
CyberPsychology \& Behavior, 10(6), 805-812. doi:10.1089/ cpb.2007.9950

Ivarsson, M., Anderson, M., Åkerstedt, T., \& Lindblad, F. (2009). Playing a violent television game affects heart rate variability. Acta Paediatrica, 98(1), 166-172. doi:10.1111/j.16512227.2008.01096.x

Kim, G. Y., Ahn, C. S., Jeon, H. W., \& Lee, C. R. (2012). Effects of the use of smartphones on pain and muscle fatigue in the upper extremity. Journal of Physical Therapy Science, 24(12), 1255-1258. doi:10.1589/jpts.24.1255

Ko, K., Kim, H. S., \& Woo, J. H. (2013). The study of muscle fatigue and risks of musculoskeletal system disorders from text inputting on a smartphone. Journal of the Ergonomics Society of Korea, 32(3), 273-278. doi:10.5143/JESK.2013.32.3.273

Lanaj, K., Johnson, R. E., \& Barnes, C. M. (2014). Beginning the workday yet already depleted? Consequences of late-night smartphone use and sleep. Organizational Behavior and Human Decision Processes, 124(1), 11-23. doi:10.1016/j. obhdp.2014.01.001

Lashley, F. R. (2003). A review of sleep in selected immune and autoimmune disorders. Holistic Nursing Practice, 17, 65-80.

Léger, D., Partinen, M., Hirshkowitz, M., Chokroverty, S., Touchette, E., \& Hedner, J. (2010). Daytime consequences of insomnia symptoms among outpatients in primary care practice: EQUINOX international survey. Sleep Medicine, 11(10), 999-1009. doi:10.1016/j.sleep.2010.04.018

Lemola, S., Perkinson-Gloor, N., Brand, S., Dewald-Kaufmann, J. F., \& Grob, A. (2015). Adolescents' electronic media use at night, sleep disturbance, and depressive symptoms in the smartphone age. Journal of Youth and Adolescence, 44(2), 405-418. doi:10.1007/s10964-014-0176-x

Li, C. H. (2015). Confirmatory factor analysis with ordinal data: Comparing robust maximum likelihood and diagonally weighted least squares. Behavior Research Methods, 3, 1-14. doi:10.3758/s13428-015-0619-7

Li, J., Lepp, A., \& Barkley, J. E. (2015). Locus of control and cell phone use: Implications for sleep quality, academic performance, and subjective well-being. Computers in Human Behavior, 52, 450-457. doi:10.1016/j.chb.2015.06.021

Li, S., Jin, X., Wu, S., Jiang, F., Yan, C., \& Shen, X. (2007). The impact of media use on sleep patterns and sleep disorders among school-aged children in China. Sleep, 30(3), 361-367. doi:10.1093/sleep/30.3.361

Lin, Y. H., Chang, L. R., Lee, Y. H., Tseng, H. W., Kuo, T. B., \& Chen, S. H. (2014). Development and validation of the Smartphone Addiction Inventory (SPAI). PLoS One, 9(6), e98312. doi:10.1371/journal.pone.0098312

Liu, D., Kirschner, P. A., \& Karpinski, A. C. (2017). A meta-analysis of the relationship of academic performance and social network site use among adolescents and young adults. Computers in Human Behavior, 77, 148-157. doi:10.1016/ j.chb.2017.08.039

Magnavita, N., \& Garbarino, S. (2017). Sleep, health and wellness at work: A scoping review. International Journal of Environmental Research \& Public Health, 14(11), 1347. doi:10.3390/ ijerph14111347

Moon, J. H., Lee, M. Y., \& Moon, N. J. (2014). Association between video display terminal use and dry eye disease in school children. Journal of Pediatric Ophthalmology and Strabismus, 51(2), 87-92. doi:10.3928/01913913-2014 0128-01 
Muthén, L. K., \& Muthén, B. O. (1998-2012). Mplus user's guide (7th ed.). Los Angeles, CA: Muthén \& Muthén.

Nagane, M., Suge, R., \& Watanabe, S. I. (2016). Time or retiring and sleep quality may be predictors of academic performance and psychosomatic disorder in university students. Biological Rhythm Research, 47(2), 329-337. doi:10.1080/09291016. 2015.1126076

Park, S., Cho, M. J., Chang, S. M., Bae, J. N., Jeon, H. J., Cho, S. J., Kim, B. S., Chung, I. W., Ahn, J. H., Lee, H. W., \& Hong, J. P. (2010). Relationships of sleep duration with sociodemographic and health-related factors, psychiatric disorders and sleep disturbances in a community sample of Korean adults. Journal of Sleep Research, 19(4), 567-577. doi:10.1111/ j.1365-2869.2010.00841.x

Perkinson-Gloor, N., Lemola, S., \& Grob, A. (2013). Sleep duration, positive attitude toward life, and academic achievement: The role of daytime tiredness, behavioral persistence, and school start times. Journal of Adolescence, 36(2), 311-318. doi:10.1016/j.adolescence.2012.11.008

Prather, A. A., Puterman, E., Epel, E. S., \& Dhabhar, F. S. (2014). Poor sleep quality potentiates stress-induced cytokine reactivity in postmenopausal women with high visceral abdominal adiposity. Brain Behavior \& Immunity, 35(1), 155-162. doi:10.1016/j.bbi.2013.09.010

Schulenberg, J., Maggs, J. L., \& Hurrelmann, K. (1997). Health risks and developmental transitions during adolescence. Cambridge, UK: Cambridge University Press.

Smick, K. (2014). Guarding your patient's eyes for harmful light: Part one: The importance of education. Review of Optometry, 151, 26-28.

Strasburger, V. C. (2009). Why do adolescent health researchers ignore the impact of the media? Journal of Adolescent Health, 44(3), 203-205. doi:10.1016/j.jadohealth.2008.12.019

Tao, F. B., Hu, C. L., Sun, Y. H., \& Hao, J. H. (2008). The development and application of Multidimensional Sub-Health Questionnaire of adolescents (MSQA). Chinese Journal of Disease Control \& Prevention, 12, 309-314.

Waller, E. A., Bendel, R. E., \& Kaplan, J. (2008). Sleep disorders and the eye. Mayo Clinic Proceedings, 83(11), 1251-1261. doi: $10.4065 / 83.11 .1251$

Wang, J., Wang, H., Gaskin, J., \& Wang, L. (2015). The role of stress and motivation in problematic smartphone use among college students. Computers in Human Behavior, 53, 181-188. doi:10.1016/j.chb.2015.07.005 